\title{
The Shark HoxN cluster is homologous to the Human HoxD cluster
}

\author{
Sonja J. Prohaska ${ }^{\ddagger}$, , Claudia Fried ${ }^{\ddagger}, \dagger$, Chris T. Amemiya ${ }^{\oplus}$, \\ Frank H. Ruddle ${ }^{\otimes}$, GÜnter P. Wagner ${ }^{\Uparrow}$, And Peter F. \\ STADLER ${ }^{\ddagger \dagger, \dagger}$ \\ ‡Bioinformatik, Institut für Informatik, \\ Universität Leipzig, Kreuzstraße 7b, D-04103 Leipzig, Germany
}

${ }^{\dagger}$ Institut für Theoretische Chemie und Molekulare Strukturbiologie Universität Wien, Währingerstraße 17, A-1090 Wien, Austria

\author{
${ }^{\oplus}$ Virginia Mason Research Center, Benaroya Research Institute, \\ Molecular Genetics Dept. 1201 Ninth Avenue, Seattle, WA 98101 USA \\ ${ }^{\otimes}$ Department of Department of Cellular and Developmental Biology \\ Yale University, New Haven, CT, USA \\ 『Department of Ecology and Evolutionary Biology \\ Yale University, New Haven, CT, USA
}

${ }^{\S}$ The Santa Fe Institute, 1399 Hyde Park Road, Santa Fe, NM 87501, USA

*Address for correspondence:

Peter F. Stadler, Bioinformatik, Institut für Informatik, Universität Leipzig, Kreuzstraße 7b, D-04103 Leipzig, Germany. Phone: ++49 341149 5120; Fax: ++49 341149 5119; Email: peter.stadler@bioinf.uni-leipzig.de.

\begin{abstract}
.
The statistical analysis of phylogenetic footprints in the two known horn shark Hox clusters and the four mammalian clusters shows that the shark HoxN cluster is HoxD-like. This finding implies that the most recent common ancestor of jawed vertebrates had at least four Hox clusters, including those which are orthologous to the four mammalian Hox clusters.
\end{abstract}

Keywords. Hox clusters, phylogenetic footprints 


\section{Introduction}

Hox genes code for homeodomain containing transcription factors which are homologous to the genes in the Drosophila homeotic gene clusters (McGinnis and Krumlauf, 1992). Vertebrates, in contrast to all invertebrates examined, have multiple Hox gene clusters that presumably have arisen from a single ancestral cluster in the most recent common ancestor of chordates, i.e. amphioxus and vertebrates (Garcia-Fernández and Holland, 1994; Kappen et al., 1989). The timing of the Hox cluster duplication events in vertebrate phylogeny is still somewhat unclear. The most popular hypothesis is that the common ancestor of sharks and bony fish (which also include the land vertebrates such as human and mouse) had four clusters homologous to the mammalian ones. (Holland and Garcia-Fernandez, 1996). To test this idea, two nearly complete Hox clusters have recently been isolated and sequenced, called $N$ and $M$ (Kim et al., 2000). While the $M$ cluster is clearly homologous to the human HoxA cluster, it was more difficult to assign the homology to the HoxN cluster. In the original description HfHoxN was identified as homologous to the human HoxD cluster, but there is also evidence consistent with homology to the HoxC cluster (Málaga-Trillo and Meyer, 2001).

\section{Materials and Methods}

In this contribution we perform a statistical analysis of conserved non-coding sequences utilizing a new software called tracker (Prohaska et al., 2003). This program is based on BLAST (Altschul et al., 1990) for the initial search of all pairs of input sequences. Comparisons are (optionally) restricted to homologous intergenic regions. The resulting list of pairwise sequence alignments is then assembled into groups of partially overlapping regions that are subsequently passed through several filtering steps. Individual phylogenetic footprints (PFs) are defined in Tagle's original paper (Tagle et al., 1988) as blocks of at least 6bp of DNA sequence that is $100 \%$ conserved in taxa that have an additive evolutionary time of 250 million years. PFs are considered to be putative transcription factor binding sites. Typically tracker detects clusters of such footprints which are termed cliques. The decomposition of cliques into individual footprints is often ambiguous. Our statistical analysis below is therefore based on the total length of significantly homologous non-coding sequence fragments between pairs of clusters. This measure is roughly proportional to the number of individual footprints. Homologous footprints are necessarily co-linear (disregarding the possibility of local transpositions or inversions which cannot be resolved with the present analysis method due to the highly diverged sequence outside the footprint clusters). Non-colinear tracker-hits are therefore disregarded (marked by $x$ in the supplemental material).

The tracker program produces alignments of the footprint cliques using dialign (Morgenstern, 1999). These are padded with "gap" characters in those sequences that do not take part in a particular clique and then concatenated. The resulting "alignment" is sparse in the sense that the "gap" character is the most frequent letter. The reconstruction of phylogenies from such a dataset has to take three complications into account: (1) gene loss will cause almost certainly the loss of all the the associated 
regulatory sequences. In the extreme case, presence-absence data of footprints might just reflect that presence-absence pattern of the genes. (2) We cannot expect to have detected all footprints in all species. (3) Gain and loss of footprints are not symmetric processes: in fact footprint loss is much easier than the de novo creation. These complications can be circumvented by considering only mutations within conserved non-coding regions, i.e., within the footprint cliques detected by the tracker program. The distance of two clusters is therefore derived from the frequency of mutations within cliques that are shared by the two clusters. Technically, this amounts to treating "gaps" as missing data rather than as an additional character state.

We use different distance-based and parsimony-type approaches here: Neighbor joining method (Saitou and Nei, 1987) (implemented in the phylip package, version 3.6) (Felsenstein, 1989), the canonical split decomposition (Bandelt and Dress, 1992), Buneman trees (Buneman, 1971), parsimony splits and P-trees (Bandelt and Dress, 1993). With the exception of NJ these methods are implemented in the splitstree package (version 3.1) (Huson, 1998). The split-based methods are particularly suitable for our purposes because they are known to be very conservative in that they tend to produce multifurcations rather than poorly supported edges (Semple and Steel, 2003). In addition we use MacClade 4.0 (Maddison and Maddison, 2000) for standard maximum parsimony analysis.

The following sequences are used for the analysis: Shark (Heterodontus francisci): M-cluster HfM = AF479755, N-cluster HfN = AF224263; Human (Homo sapies): $\mathrm{HsA}=\mathrm{AC004080.2 \textrm {rc }}+\mathrm{AC010990}$ [201-6508] $\mathrm{rc}+\mathrm{AC004079}$ [75001-end]rc, HsB = NT_010783 [931646-1263780]rc, HsC = NT_009563 [580371-708054]rc, HsD = NT_037537 [4075338-end]. Rat (Rattus norvegicus): RnA = NW_043751 [910030-1194462]rc, $\mathrm{RnB}=$ NW_042671 [264022-581839], RnC = NW_044048 [722873-1060956] RnD = NW_042732 [1061702-1217610]rc. Fugu (Takifugu rubripes) sequences are taked from the Fugu database DOE Joint Genome Institute: TrAa = scaffold_47 of release_3.0, $\operatorname{Tr} \mathrm{Ab}=$ scaffold 1874 of release $2.0, \operatorname{TrD}=$ scaffold_3959+scaffold_214[160440-end]rc. Here "rc" means that the reverse complement of the database entry has been used (after extracting the indicated interval).

\section{Results}

A comparison of the protein sequences of the shark HoxN cluster with mammalian Hox protein sequences is consistent with $D$-likeness, although the data in Table 1 do not show an unambiguous picture. In particular, the HoxD proteins are not always the ones with the highest degree of sequence identity, see Table 1. In a similar vein, the analysis of Hox genes and of genes linked to the Hox clusters such as collagens does not yield an unambiguous picture for the branching order of the four mammalian Hox clusters (Bailey et al., 1997).

Let us now turn to the analysis of the conserved parts of the non-coding sequences. Table 2 summarizes the results of pairwise comparisons of shark and human (or rat) Hox clusters. It should be noted that the sequence of the shark HoxN cluster is incomplete, spanning only the sequence from evx to (almost) Hox-4. There is a particularly high conservation of non-coding sequences between shark HoxM and 
Table 1. Best correspondences of Hox proteins with the HoxN sequence of the hornshark. Numbers are percentages of sequence identities in protein alignments obtained with clustalw (Thompson et al., 1994). Italics and sans serif fonts indicate that the best match is by the human or rat sequence, respectively. Empty fields indicate that there was no good match, a dash - denotes genes that do not exist in the mammalian Hox clusters.

\begin{tabular}{|l|cccccccc|}
\hline Cluster & evx & 13 & 12 & 11 & 10 & 9 & 8 & 5 \\
\hline $\mathrm{A}$ & & 70 & - & 57 & & 63 & - & 53 \\
$\mathrm{~B}$ & - & & - & - & - & & 68 & 48 \\
$\mathrm{C}$ & - & & 48 & 54 & & 63 & 69 & 44 \\
$\mathrm{D}$ & 81 & 68 & 48 & 57 & 69 & 61 & 71 & - \\
\hline
\end{tabular}

mammalian HoxA sequences in the range from Hox-4 to Hox-1. As a consequence, the counts for $\operatorname{HoxN}$ are significantly smaller. In table 2 we therefore display the data for both the full length clusters and the restriction to the region between evx and Hox-4. The total length of sequences conserved between shark and mammalian clusters in this region is comparable between HfM and HfN.

The homology of the shark HoxM and the mammalian HoxA clusters is obvious from these data. For the $\operatorname{HoxN}$ sequence we find little distinction when counting colinear cliques and only a moderate signal in the numbers of co-linear clusters. The total length of the conserved regions, however, is more than twice as large with HoxD than with $H_{0 x C}$ and about $50 \%$ longer in $H_{0 x D}$ compared to HoxA. The location and distribution of the footprint cliques, Fig. 1 also strongly argues for a homology with HoxD rather than $H o x C$.

Table 2. Pairwise comparison of non-coding sequences in the shark Hox clusters with mammalian Hox clusters. In addition we report the comparison with preliminary HoxC and $\operatorname{HoxD}$ cluster sequences (obtained from version 3.0 of the Fugu database (DOE Joint Genome Institute; Aparicio et al., 2002); see (Prohaska et al., 2003) for details). For the duplicated Hox $A$ and HoxB clusters we list the number and lengths of cliques that hornshark shares with at least one of duplicates.

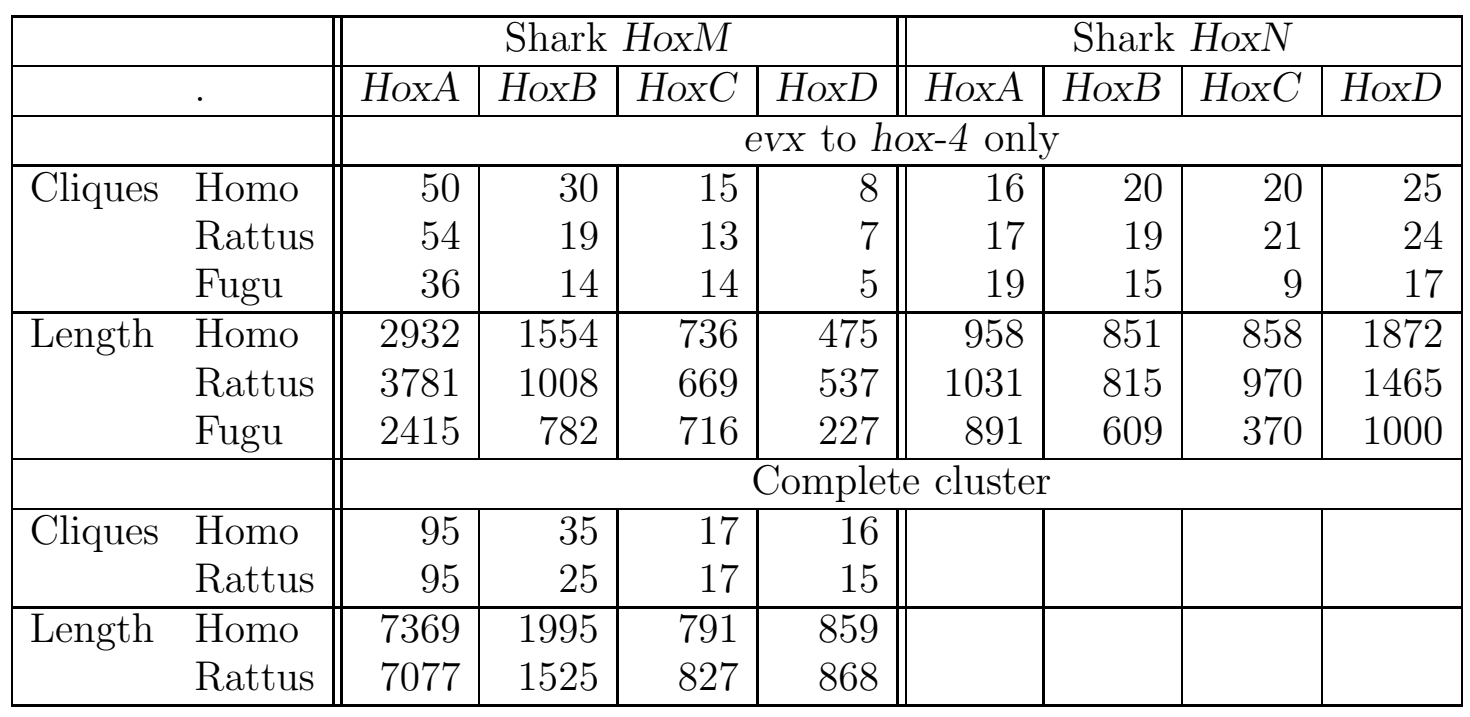




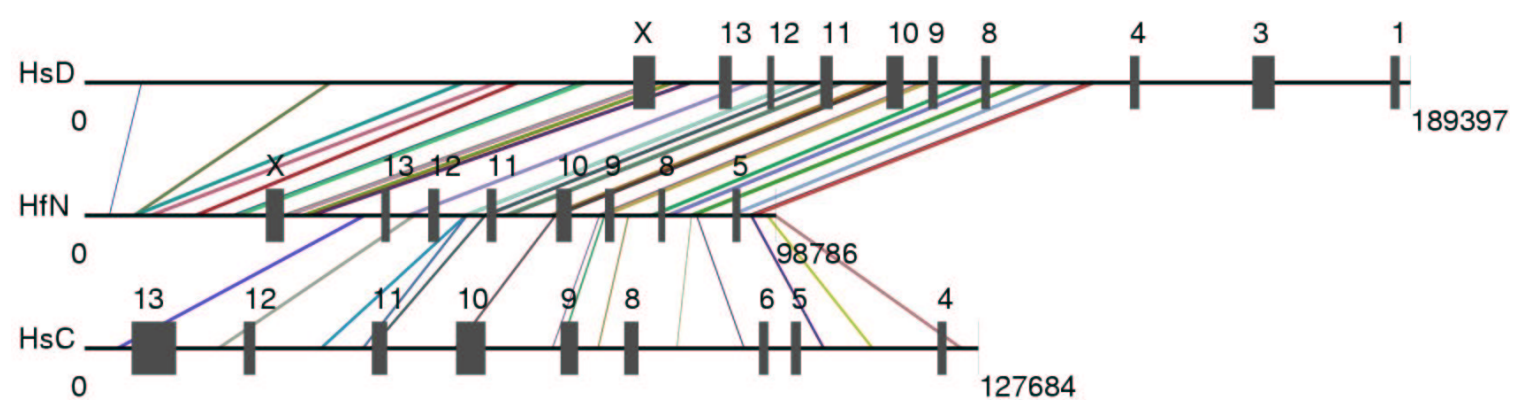

Figure 1. Overview of the phylogenetic footprint cliques produced by tracker for the comparison of the horn shark HoxN sequence (HfN) and the human HoxC (HsC) and HoxD (HsD) sequences, respectively. X denotes the Evx genes.

A comparison of HfHoxN with the fugu (Takifugu rubripes) HoxC $\alpha$ and HoxD sequences also places HfHoxN with the $D$ rather than $C$ cluster. These data must be interpreted with caution: (i) The Fugu sequences are preliminary constructs combining two or three scaffolds and hence not complete. (ii) Even though the current version 3.0 of the Fugu genome database (DOE Joint Genome Institute) does not contain evidence of a $C \beta$ cluster, it is most likely that the teleost $C$ cluster was duplicated since the zebrafish (Danio rerio) does have both a $\mathrm{Hox} C \alpha$ and a $H o x C \beta$ cluster (Amores et al., 1998). The duplication event might have caused the additional loss of a substantial number of footprints. Nevertheless, we find that the counts for the hornshark-pufferfish comparisons are similar to the shark-mammal comparisons.

The sensitivity of the tracker method is increased by including more sequences. In particular, homologous footprints can be identified between two sequences even if they do not yield a significant signal when the two sequences are compared directly. We have therefore performed a complete analysis of both shark clusters and all four human Hox clusters. The supplemental material lists all footprint cliques in the range from evx to hox-1 that appear in at least one shark and at least one human cluster. The statistics of the conserved regions between clusters is summarized in Table 3.

Treating phylogenetic footprint cliques as presence/absence characters in a parsimony framework also supports the hypothesis that HfHoxN is more closely related to $H_{s H o x D}$ than to $H_{s} H o x C$. The tree $((A, M),(C,(D, N))$ is seven steps shorter than $((\mathrm{A}, \mathrm{M}),((\mathrm{C}, \mathrm{N}), \mathrm{D})$ (tree length $=402, C I=0.57, R I=0.18)$. This result is based on the assumption of a $((A, B),(C, D))$ scenario, which is favored from the analysis of Hox sequences, see for instance (Amores et al., 1998). The alternative, which is supported by the analysis of genes linked to the Hox clusters by Bailey et al. (1997), leads to considerably shorter trees. The tree $(((A, M),(D, N)),(C, B))$ has 374 steps $(C I=0.62$, $R I=0.43)$ compared to $(((\mathrm{A}, \mathrm{M}), \mathrm{D}),((\mathrm{C}, \mathrm{N}), \mathrm{B}))$, which is 44 steps longer. While we do not want to get into the question of which cluster phylogeny applies to the human Hox clusters here, we just want to note that in either scenario a tree with HfHoxN most closely related to $H_{s} H_{o x} D$ is more parsimonious than any other phylogenetic position of HfHoxN (data not shown).

These data clearly indicate that the shark $\operatorname{HoxN}$ cluster is $H o x D$-like at least as far as the non-coding sequences are concerned. In fact, based on total size of the footprints 
Table 3. Comparison of phylogenetic footprints from a tracker run of both shark and all four human clusters. Only co-linear cliques in range between evx and hox-1 are counted. The data contain six cliques $(484,485,486,513,514,515$ in the supplement) of which at most three are consistent with co-linearity. These are counted with a weight $1 / 2$.

\begin{tabular}{|c|c|c|c|c|c|c|c|c|}
\hline & \multicolumn{4}{|c|}{ Shark HoxM } & \multicolumn{4}{|c|}{ Shark HoxN } \\
\hline & HoxA & HoxB & $\mathrm{HoxC}$ & HoxD & HoxA & HoxB & $\mathrm{HoxC}$ & HoxD \\
\hline & \multicolumn{8}{|c|}{ evx to hox-4 } \\
\hline Cliques Homo & 47 & 21 & 13 & 9 & 15 & 10 & 20 & 25 \\
\hline $\begin{array}{ll}\text { Length } & \text { Homo }\end{array}$ & 3847 & 1905 & 646 & 1065 & 1728 & 961 & 1148 & 1995 \\
\hline & \multicolumn{8}{|c|}{ Complete cluster } \\
\hline Cliques Homo & 79 & 24 & 13 & 16 & & & & \\
\hline Length & 6937 & 2268 & 1142 & 1598 & & & & \\
\hline
\end{tabular}

that are shared between clusters, the next candidate would be the mammalian $A$ cluster, not the $C$-cluster as proposed in (Málaga-Trillo and Meyer, 2001).

To test whether $\operatorname{HfHoxN}$ is a true homologue of the mammalian HoxD clusters we consider the co-occurrences of the 49 footprint cliques that are present in $\operatorname{HfHoxN}$, Table 4. In particular, there are 14 cliques that HfHoxN shares uniquely with human $H_{s H o x D}$, compared to 10 cliques shared with the HoxC cluster and only 5 to 6 that are only shared with the HoxA and HoxB clusters, respectively. On the other hand, about $30 \%$ of the footprints are shared between HfHoxD, one of the Human clusters and at least one other mammalian cluster. The footprints shared between HoxN and either HoxA or HoxB are to $90 \%$ also shared with HoxM. Together, these data strongly suggest that HfHoxN is not only most similar to the mammalian HoxD clusters but is a true homologue.

Table 4. Footprints shared between shark $\operatorname{HoxN}$, one of the four human clusters, and other Hox clusters.

There are 49 footprint cliques with a total length of 3042nt involving HfHoxN. We obtain two different counts for the number of clusters exclusively shared between HfHoxN and $H_{s} H o x B$ arising from the mutually incompatible cliques marked with o in the supplement.

\begin{tabular}{|l||r|r|r|r|}
\hline \multicolumn{1}{|c||}{} & \multicolumn{4}{c|}{ shared with the human cluster } \\
& Hox $A$ & HoxB & HoxC & HoxD \\
\hline exclusively & 5 & $5-6$ & 10 & 14 \\
\hline cliques & 234 & $\sim 200$ & 267 & 640 \\
length & 8 & 7 & 9 & 21 \\
precentage & 8 & \multicolumn{4}{|c|}{} \\
\hline plus at least one other mammalian cluster \\
\hline cliques & 9 & 5 & 10 & 11 \\
length & 1166 & 741 & 850 & 1051 \\
percentage & 38 & 24 & 28 & 35 \\
\hline plus HfM & \multicolumn{4}{|}{} \\
\hline cliques & 7 & 4 & 5 & 5 \\
length & 149 & 38 & 248 & 359 \\
percentage & 87 & 95 & 71 & 66 \\
\hline
\end{tabular}


Further evidence for this claim can be obtained from the phylogenetic analysis of the combined footprint cliques of the four mammalian clusters for either human or rat together with the two available shark sequences. Both distance-based, Fig. 2 and parsimony-based methods, Fig. 3, agree on this interpretation. We have chosen a variety of split-based algorithms for this analysis because these techniques are known to produce multifurcations rather than poorly supported edges. For comparison standard neighbor-joining trees are shown in Fig. 2.

All data presented in Figs. 2 and 3 either support the conclusion that the shark HoxN cluster is homologous with mammalian HoxD cluster or are at least consistent with this conclusion (whenever the HfHoxN-HoxD node is a multifurcation).

\section{Discussion}

The evidence presented in this paper supports the original hypothesis, namely that the shark HoxN cluster is orthologous to the mammalian HoxD cluster (Kim et al., 2000). The method employed is novel, namely to use the distribution and extent of non-coding sequences for phylogenetic inferences. Below we discuss the implications of the present finding for our understanding of Hox cluster evolution in vertebrates.

Conserved non-coding sequences have long been used to find candidate cis-regulatory elements, see (Duret and Bucher, 1997) for a review. Identification of putative cisregulatory sequences requires long stretches of sequence from distantly related species (Tagle et al., 1988) or a set of species which have sufficient additive divergence among them (Sumiyama et al., 2001). More recently this method has been used to trace the non-coding sequence divergence after HoxA cluster duplication in teleosts (Chiu et al., 2002). In this paper it has been shown that non-coding sequences can remain highly conserved in the absence of Hox gene cluster duplication, as documented between the shark HoxM and the mammalian HoxA cluster (see also this paper). Hence it is possible to treat the loss and the acquisition of conserved non-coding sequences as potentially apomorphic characters. Thus they contain phylogenetic information. The congruence between the structural and coding sequence evidence and the comparison on non-coding sequence conservation for HoxM and HoxA cluster validates this assumption. In the case of the shark HoxN cluster the evidence from coding sequence and structural organization is less strong and we thus rely on the evidence from non-coding sequence conservation. While the signal is still not as strong as for the HoxM each analysis is at least consistent and in many cases positively supportive of orthology between shark HoxN and mammalian HoxD cluster.

The conclusion that both the shark HoxM as well as the HoxN clusters are directly orthologous to the mammalian HoxA and HoxD clusters, respectively, has important implications for the history of Hox cluster duplications. It follows that the most recent common ancestor of cartilaginous fish and the bony fish clade (which includes mammals) had at least four Hox clusters orthologous to the four mammalian Hox clusters. It is thus likely that sharks have two more clusters than those currently described. This evidence also confirms the hypothesis of Peter Holland that the four cluster situation typical for most major gnathostome lineages has arisen before the most recent common ancestor of all recent gnathostomes (Garcia-Fernández and 


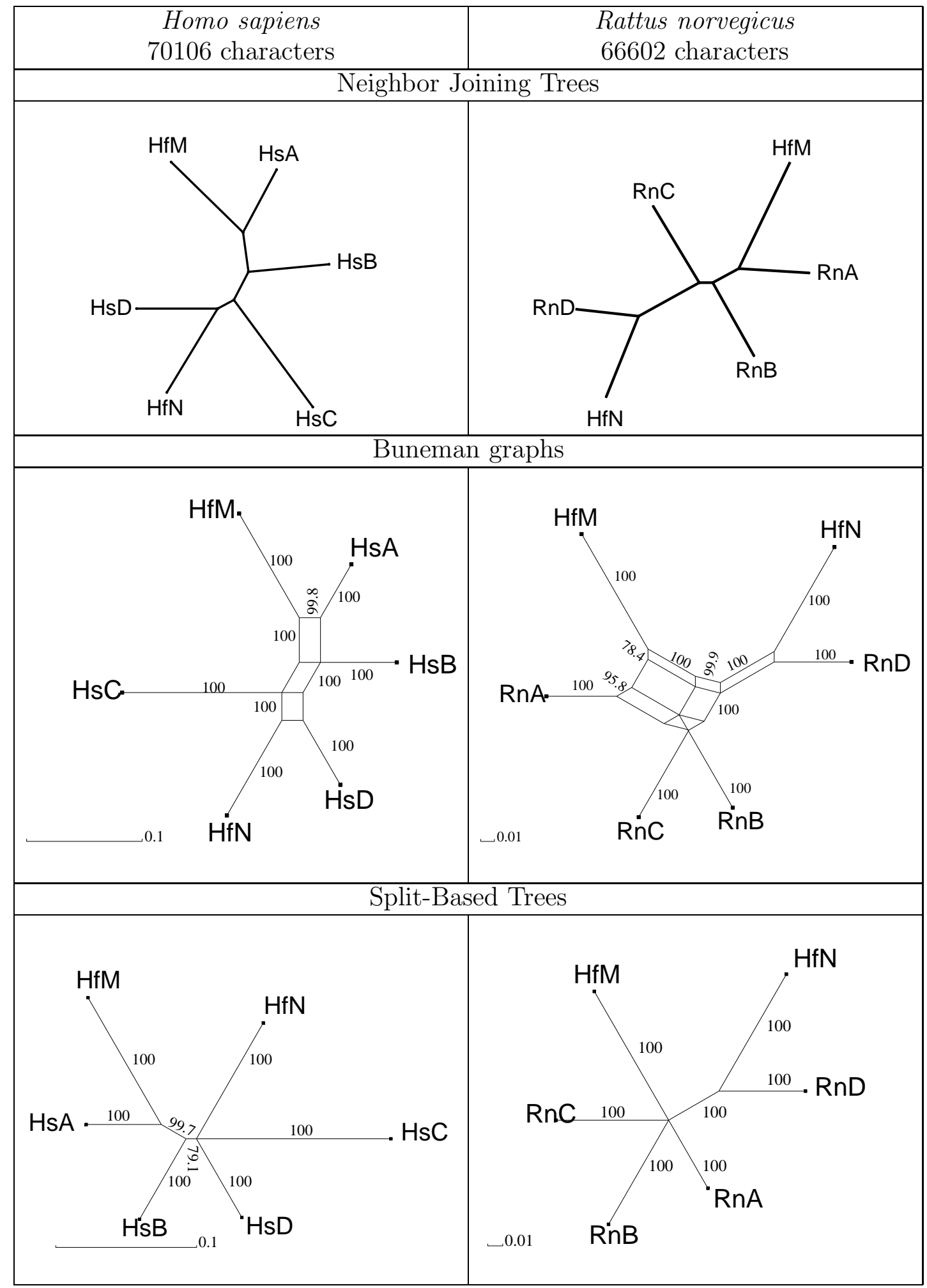

Figure 2. Distance-based phylogenies of shark and mammalian Hox clusters. Neighbor joining trees (Saitou and Nei, 1987) are computed using Felsenstein's phylip package (version 3.6). Buneman graphs representing the canonical decomposition of the distance function and the split-based Buneman trees are computed using Daniel Huson's splitstree package, version 3.1, (Huson, 1998). 


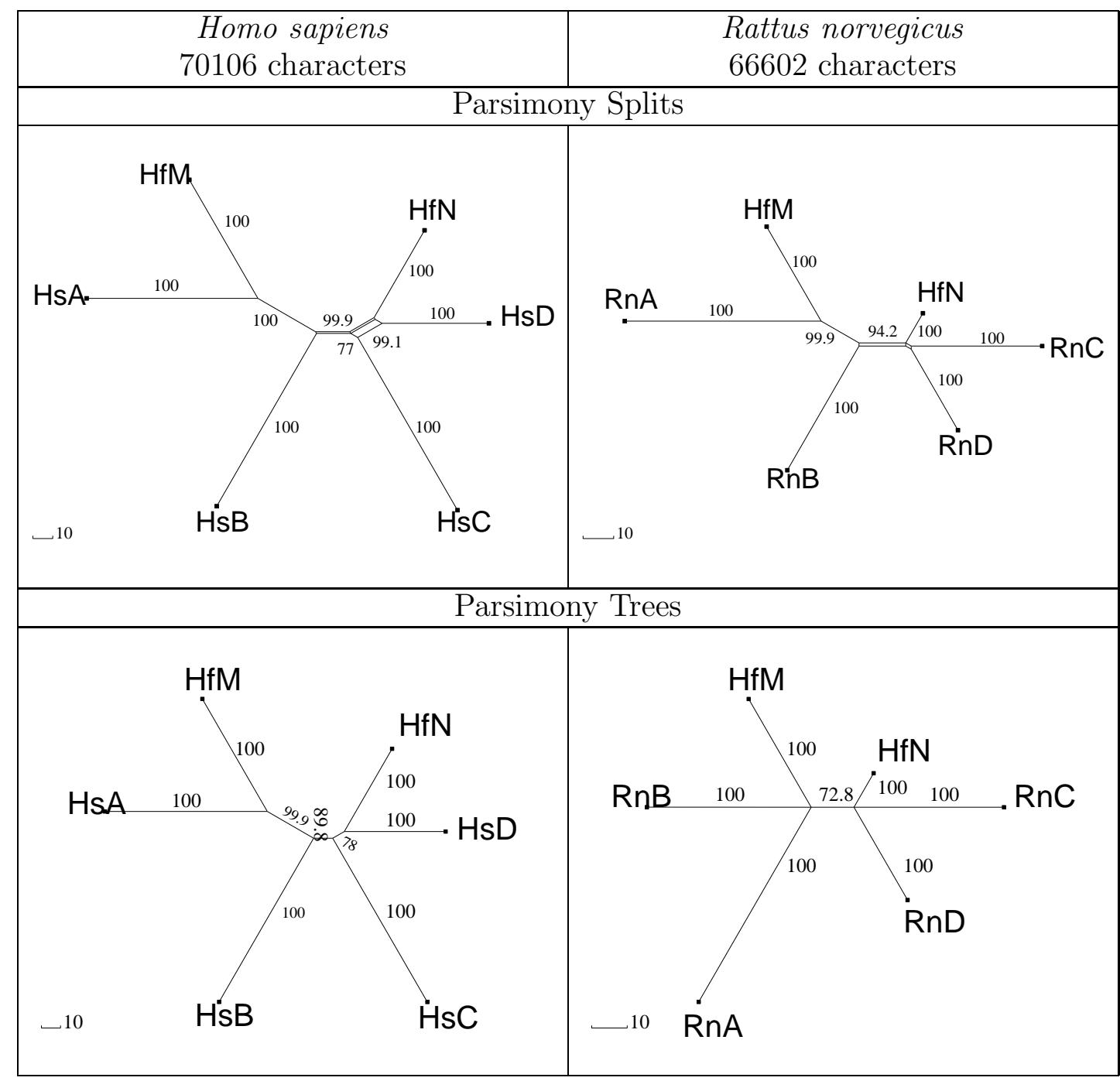

Figure 3. Parsimony-based phylogenies of shark and mammalian Hox clusters computed using splitstree, version 3.1, (Huson, 1998).

Holland, 1994; Holland et al., 1994). Of course this result does not guarantee that all gnathostome lineages in fact have at least four Hox clusters since clusters can be lost. This can happen in particular soon after the duplication, which might have occurred shortly before the split between the shark and mammalian lineages.

Acknowledgments. Funding for this research is gratefully acknowledged: DFG Bioinformatics Initiative BIZ-6/1-2 to SJP, CF, and PFS; NSF IBN-9905403 to FR and GPW;

\section{References}

Altschul S.F., Gish W., Miller W., Myers E.W., and Lipman D.J., 1990. Basic local alignment search tool. J. Mol. Biol. 215:403-410. 
Amores A., Force A., Yan Y.L., Joly L., Amemiya C., Fritz A., Ho R.K., Langeland J., Prince V., Wang Y.L., et al., 1998. Zebrafish hox clusters and vertebrate genome evolution. Science 282:1711-1714.

Aparicio S., Chapman J., Stupka E., Putnam N., Chia J.M., Dehal P., Christoffels A., Rash S., Hoon S., Smit A., et al., 2002. Whole-genome shotgun assembly and analysis of the genome of Fugu rubripes. Science 297:1301-1310.

Bailey W.J., Kim J., Wagner G., and Ruddle F.H., 1997. Phylogenetic reconstruction of vertebrate Hox cluster duplications. Mol. Biol. Evol. 14:843-853.

Bandelt H.J. and Dress A.W.M., 1992. A canonical decomposition theory for metrics on a finite set. Adv. math. 92:47.

Bandelt H.J. and Dress A.W.M., 1993. A relational approach to split decomposition. In Information and Classification, editors Opitz O., Lausen B., and Klar R., pages 123-131. Springer-Verlag, Berlin.

Buneman P., 1971. The recovery of trees from measures of dissimilarity. In Mathematics and the Archeological and Historical Sciences, editors Hodson F.R., Kendall D.G., and Tautu P., pages 387-395. Edinburgh University Press, Edinburgh, UK.

Chiu C.h., Amemiya C., Dewar K., Kim C.B., Ruddle F.H., and Wagner G.P., 2002. Molecular evolution of the HoxA cluster in the three major gnathostome lineages. Proc. Natl. Acad. Sci. USA 99:5492-5497.

DOE Joint Genome Institute, 2002. Fugu genome database.

version 2.0: http://genome.jgi-psf .org/fugu3/fugu3.home.html, version 3.0: http://genome.jgi-psf .org/fugu6/fugu6.home.html.

Duret L. and Bucher P., 1997. Searching for regulatory elements in human noncoding sequences. Curr. Opin. Struct. Biol. 7:399-406.

Felsenstein J., 1989. Phylip - phylogeny inference package (version 3.2). Cladistics 5:164-166.

Garcia-Fernández J. and Holland P.W., 1994. Archetypal organization of the amphioxus hox gene cluster. Nature 370:563-566.

Holland P.W. and Garcia-Fernandez J., 1996. Hox genes and chordate evolution. Dev. Biol. 173:382-395.

Holland P.W.H., Garcia-Fernández J., Williams N.A., and Sidow A., 1994. Gene duplication and the origins of vertebrate development. Development (Suppl.):125133.

Huson D.H., 1998. Splitstree: analyzing and visualizing evolutionary data. Bioinformatics 14:68-73.

Kappen C., Schughart K., and Ruddle F.H., 1989. Two steps in the evolution of antennapedia-class vertebrate homeobox genes. Proc. Natl. Acad. Sci. USA 86:5459-5463.

Kim C.B., Amemiya C., Bailey W., Kawasaki K., Mezey J., Miller W., Minosima S., Shimizu N., P. W.G., and Ruddle F., 2000. Hox cluster genomics in the horn shark, heterodontus francisci. Proc. Natl. Acad. Sci. USA 97:1655-1660.

Maddison D.R. and Maddison W.P., 2000. MacClade 4: Analysis of Phylogeny and Character Evolution. Sinauer Associates, Sunderland, Massachusetts. E-book and computer program.

Málaga-Trillo E. and Meyer A., 2001. Genome duplications and accelerated evolution of Hox genes and cluster architecture in teleost fishes. Amer. Zool. 41:676-686. 
McGinnis W. and Krumlauf R., 1992. Homeobox genes and axial patterning. Cell 68:283-302.

Morgenstern B., 1999. DIALIGN 2: improvement of the segment-to-segment approach to multiple sequence alignment. Bioinformatics 15:211-218.

Prohaska S., Fried C., Flamm C., Wagner G., and Stadler P.F., 2003. Surveying phylogenetic footprints in large gene clusters: Applications to Hox cluster duplications. Mol. Phyl. Evol. Submitted; SFI preprint \#03-02-011.

Saitou N. and Nei M., 1987. The neighbor-joining method: a new method for reconstructing phylogenetic trees. Mol Biol. Evol. 4:406-425.

Semple C. and Steel M., 2003. Phylogenetics. Oxford University Press, Oxford UK.

Sumiyama K., Kim C., and Ruddle F.H., 2001. An efficient cis-element discovery method using multiple sequence comparisons based on evolutionary relationships. Genomics 71:260-262.

Tagle D.A., Koop B.F., Goodman M., Slightom J.L., Hess D.L., and Jones R.T., 1988. Embryonic epsilon and gamma globin genes of a prosimian primate (galago crassicaudatus). Nucleotide and amino acid sequences, developmental regulation and phylogenetic footprints. J. Mol. Biol. 203:439-455.

Thompson J.D., Higgs D.G., and Gibson T.J., 1994. CLUSTALW: improving the sensitivity of progressive multiple sequence alignment through sequence weighting, position specific gap penalties, and weight matrix choice. Nucl. Acids Res. 22:46734680 . 
